\title{
SIZE AND STRUCTURE OF THE NARROW-LINE REGION OF QUASARS ${ }^{1}$
}

\author{
Nicola Bennert, ${ }^{2}$ Heino Falcke, ${ }^{3}$ Hartmut Schulz, ${ }^{2}$ Andrew S. Wilson, ${ }^{4}$ and Beverley J. Wills ${ }^{5}$ \\ Received 2002 May 14; accepted 2002 June 18; published 2002 June 27
}

\begin{abstract}
We have observed the narrow-line regions (NLRs) of the seven brightest radio-quiet Palomar-Green (or Bright Quasar Survey) quasars $(z<0.5)$ with the Wide Field Planetary Camera 2 on board the Hubble Space Telescope $(H S T)$. Linear-ramp filters were used to image the [O III] $\lambda 5007$ line emission with $0.0455-0$ ".1 pixel resolution. We find that the NLRs are very compact with typical extents of $2^{\prime \prime}-4$ ". Two quasars show compact filamentary structures similar to Seyfert NLRs. They may be related to radio outflows. Most interestingly, when including a sample of Seyfert galaxies observed with HST, we tentatively find that the size of the NLR is proportional to the square root of the [O III] luminosity. This is comparable to the scaling found for the size of the broad-line region with continuum luminosity, which has been interpreted in terms of a constant photoionization parameter. The relation determined here connects the NLR of radio-quiet quasars and Seyfert galaxies over 3 orders of magnitude in [O III] luminosity.
\end{abstract}

Subject headings: galaxies: active — galaxies: Seyfert — galaxies: structure — quasars: emission lines — quasars: general

On-line material: color figure

\section{INTRODUCTION}

Quasars are active galactic nuclei (AGNs) in which two different regions of ionized gas can be distinguished: the broadline region (BLR) and the narrow-line region (NLR), which exhibit optical emission lines that are kinematically broadened with typical widths of $10^{3}-10^{4}$ and $10^{2}-10^{3} \mathrm{~km} \mathrm{~s}^{-1}$, respectively. The optical spectra of quasars resemble those of the less luminous Seyfert galaxies, and it is presumed that most radioquiet quasars are their more luminous counterparts. The most prominent optical emission lines of the NLR are [O III] $\lambda 5007$ (hereafter referred to simply as [O III]) and $\mathrm{H} \alpha+[\mathrm{N} \mathrm{II}] \lambda \lambda 6548$, 6584.

In the unified model of AGNs, an optically thick obscuring dust torus is envisioned to encircle the accretion disk (Antonucci 1993), leading to a so-called ionization cone formed by the anisotropic escape of ionizing photons (Schulz 1988; Pogge 1988, 1989; Storchi-Bergmann, Mulchaey, \& Wilson 1992). Thus, the NLR is expected to show a specific morphology, which is suited for an investigation using the high spatial resolution afforded by the Hubble Space Telescope (HST). Such investigations have been carried out for Seyfert galaxies, revealing highly elongated structures or ionization cones (Capetti et al. 1996; Falcke, Wilson, \& Simpson 1998).

While the NLR in Seyfert galaxies is now relatively well studied, there are no comparable studies for the NLR in quasars. By observing quasars, we can greatly extend the luminosity range in order to answer questions such as: Are quasars indeed

\footnotetext{
${ }^{1}$ Based on observations with the NASA/ESA Hubble Space Telescope, obtained at the Space Telescope Science Institute, which is operated by AURA, Inc., under NASA contract NAS 5-26555.

${ }^{2}$ Astronomisches Institut Ruhr-Universität Bochum, Universitätsstrasse 150, D-44780 Bochum, Germany; nbennert@astro.ruhr-uni-bochum.de, hschulz@ astro.ruhr-uni-bochum.de.

${ }^{3}$ Max-Planck-Institut für Radioastronomie, Auf dem Hügel 69, D-53121 Bonn, Germany; hfalcke@mpifr-bonn.mpg.de.

${ }^{4}$ Department of Astronomy, University of Maryland, College Park, MD 20742-2421; wilson@astro.umd.edu.

${ }^{5}$ Department of Astronomy and McDonald Observatory, University of Texas at Austin, RLM 15.308, C-1400, Austin, TX 78712-1083; bev@pan.as.utexas.edu.
}

just scaled-up versions of Seyfert galaxies? Does the size of the NLR scale with luminosity? Is this emission-line region possibly affected by radio jets shaping the interstellar medium, or is there a contribution from star formation?

We make a first step in this direction by presenting an HST emission-line imaging survey of a complete sample of the seven brightest (in [O III]; Boroson \& Green 1992) radio-quiet quasars from the Bright Quasar Survey (Schmidt \& Green 1983; Kellermann et al. 1989) with $z<0.5$. The sample is given in Table 1. Here we present an investigation of the NLR structure as seen in the $[\mathrm{O}$ III] line and discuss the size of the NLR as a function of luminosity.

Luminosity distances for both Seyfert galaxies and quasars were calculated by using redshifts relative to the $3 \mathrm{~K}$ background as derived with the velocity calculator provided by the NASA Extragalactic Database (NED). Throughout this Letter, we adopt a Hubble constant of $H_{0}=65 \mathrm{~km} \mathrm{~s}^{-1} \mathrm{Mpc}^{-1}$ and a homogeneous, isotropic, flat world model, which includes Einstein's cosmological constant $\Lambda$ in agreement with recent supernova measurements (Perlmutter et al. 1999): $\Sigma \Omega=1$, $\Omega_{\text {matter }}=0.35, \Omega_{\text {radiation }}=0.05$, and $\Omega_{\Lambda}=0.6$.

\section{OBSERVATION, DATA REDUCTION, AND ANALYSIS}

\subsection{HST Observations}

All quasars in our sample were observed with the Wide Field Planetary Camera 2 (WFPC2) on board HST between 2000 January and October. Except for observations of the adjacent continua, we used the linear-ramp filters (LRFs). A description of properties and data reduction of LRF data is given in Falcke et al. (1998).

For our observations, the filters and central wavelengths were chosen to be centered on the redshifted [O III] emission lines. PG $0026+129$ was the only quasar that could be imaged with the Planetary Camera and its smaller pixel scale of 0 . $0455 \mathrm{pixel}^{-1}$. The other quasar images were taken with the Wide Field Camera $\left(0^{\prime \prime} 1\right.$ pixel $\left.^{-1}\right)$. Several short integrations of a bright $\operatorname{star}(V=11-16)$ were taken in the LRF at the 
TABLE 1

DETAILS OF OBSERVATION AND REDUCTION

\begin{tabular}{|c|c|c|c|c|c|c|c|}
\hline Quasar & $\begin{array}{c}\alpha \\
(\mathrm{J} 2000)\end{array}$ & $\begin{array}{c}\delta \\
(\mathrm{J} 2000)\end{array}$ & $z^{\mathrm{a}}$ & [O III] Integration ${ }^{\mathrm{b}}$ & Continuum Integration $^{\mathrm{b}}$ & PSF Star Integration ${ }^{\mathrm{b}}$ & Subtraction Method ${ }^{c}$ \\
\hline PG $0026+129 \ldots \ldots$ & 002913.71 & +131603.83 & 0.142 & $300(8)$ & $120(2)$ & $6(2)$ & PSF star scaled to continuum \\
\hline PG $0052+251 \ldots \ldots$ & 005452.13 & +252539.33 & 0.155 & $280(5)$ & $60(4)$ & $35(4)$ & PSF star scaled to continuum \\
\hline PG $0157+001 \ldots \ldots$ & 015950.21 & +002341.55 & 0.163 & $240(6)$ & $60(4)$ & $40(4)$ & Continuum \\
\hline PG $0953+414 \ldots \ldots$ & 095652.35 & +411522.53 & 0.234 & $165(8)$ & $60(4)$ & $50(4)$ & Continuum \\
\hline PG $1012+008 \ldots \ldots$ & 101458.10 & +003205.44 & 0.187 & $400(4)$ & $\ldots$ & $60(3)$ & Scaled PSF star \\
\hline PG $1049-005 \ldots \ldots$ & 105151.46 & -005118.16 & 0.360 & $212.5(8)$ & $60(4)$ & $40(4)$ & Scaled continuum \\
\hline PG $1307+085 \ldots \ldots$ & 130947.93 & +081949.62 & 0.155 & $250(6)$ & $60(4)$ & $35(4)$ & PSF star scaled to continuum \\
\hline
\end{tabular}

Note. - Units of right ascension are hours, minutes, and seconds, and units of declination are degrees, arcminutes, and arcseconds.

${ }^{a}$ Heliocentric redshift from NED.

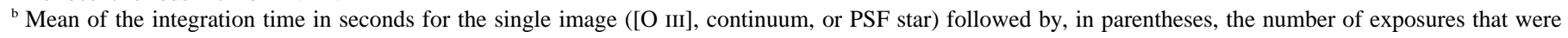
combined.

${ }^{c}$ Scaled PSF star, continuum, scaled continuum, or PSF star weighted by the flux in the continuum image.

same position on the chips as the corresponding quasar, to determine the point-spread function (PSF) for calibration purposes.

To subtract the underlying continuum, images of the adjacent continua were taken in narrowband filters (F588N: $\delta \lambda=49 \AA$, F631N: $\delta \lambda=30.9 \AA$, and F673N: $\delta \lambda=47.2 \AA$ ), at the same chip position as the LRF images. All observations were split into several integrations to allow for cosmic-ray rejection with integration times chosen to avoid saturation at the position of the central point source.

\subsection{Data Reduction and Analysis}

All exposures were bias- and dark-subtracted and, except for the LRF images, flat-field-corrected by WFPC2 pipeline processing at the Space Telescope Science Institute (STScI). For the LRFs, we used flat fields taken in nearby narrowband filters. In all cases, the different exposures were shifted (using linear interpolation as recommended for subsampled data) by aligning the central intensity peaks of the LRF, continuum, and PSF images, as determined from Gaussian-profile fitting. The pointsource calculator (as provided by STScI) was used for flux calibration. For the subtraction of the continuum and especially the contribution from the unresolved continuum source at the center of the quasar, we used either the continuum images or the PSF star (see Table 1). In one case (PG 1012+008), no continuum was detected on the continuum images, and a scaled PSF star was subtracted from the LRF image. The scaling was done such that, after subtraction, the central pixel had the same value as the average of the neighboring pixels. This avoids "holes" in the center and may lead to an underestimate of the [O III] emission. We also had to scale the "continuum" image for PG 1049-005 in the same way since the image was, by accident, not taken in a continuum wavelength range but in the middle of the emission line of [O III] $\lambda 4959$. For PG $0157+001$ and PG $0953+414$, the continua were clearly extended compared with that of the PSF star, and the continuum images were used for subtraction. In the three remaining cases (PG 0026+ 129, PG $0052+251$, and PG $1307+085)$, the continua were not extended, compared with the PSF star, and we subtracted the PSF star, scaled to the flux seen in the continuum image, because of the higher signal-to-noise ratio of the star.

To check how much galaxy line emission or continuum we might be missing by using our short-exposure continuum (or scaled PSF) images, we used broadband continuum images of the host galaxies of our quasars from Bahcall et al. (1997). The broadband images were scaled to the appropriate filter width and exposure time of our LRF images and then subtracted. We found the same [O III] flux at radii greater than 2-10 pixels, where the broadband images were not overexposed, and concluded that, indeed, the host-galaxy continuum contribution to the LRF images was negligible outside the very nucleus. We also performed surface photometry of the continuum-subtracted LRF images and compared the resulting [O III] luminosities (Table 2) with the [O III] magnitudes determined by Boroson \& Green (1992) with ground-based spectroscopy in a $6^{\prime \prime} \times 1.5$ extraction aperture. After correcting the luminosity distances for differences in the Hubble constant (a 30\% effect) and the world model ( 3\%), the difference between the HST imaging and ground-based spectroscopic data scatters within 0.25 mag, consistent with the expected spectrophotometric errors. The evidence suggests that we have not missed any significant extended flux and that our continuum subtraction is correct.

TABLE 2

EMISSION-Line FluX AND Size of THE NLRs

\begin{tabular}{|c|c|c|c|c|c|c|c|}
\hline Quasar & $\begin{array}{l}\text { Luminosity } \\
\text { Distance } \\
(\mathrm{Mpc})\end{array}$ & $\begin{array}{l}\text { Radius of } \\
\text { Aperture } \\
\text { (arcsec) }\end{array}$ & $\begin{array}{l}\text { Radius of } \\
\text { NLR } \\
\text { (pc) }\end{array}$ & $\begin{array}{c}{[\mathrm{O} \text { III }] \lambda 5007} \\
\text { Flux }^{\mathrm{a}} \\
\left(\times 10^{-14} \text { ergs s}^{-1} \mathrm{~cm}^{-2}\right)\end{array}$ & $\begin{array}{c}{[\mathrm{O} \text { III] }] \text { 入5007 }} \\
\text { Luminosity } \\
\left(\times 10^{42} \mathrm{ergs} \mathrm{s}^{-1}\right)\end{array}$ & $\begin{array}{l}\mathrm{H} \beta \text { Luminosity } \\
\left(\times 10^{42} \mathrm{ergs} \mathrm{s}^{-1}\right)\end{array}$ & $\begin{array}{c}\text { Surface Brightness }{ }^{\mathrm{b}} \\
\left(\times 10^{-17} \mathrm{ergs} \mathrm{s}^{-1} \text { pixel }^{-1}\right)\end{array}$ \\
\hline PG $0026+129$ & 708 & 0.86 & $2281 \pm 240$ & 7 & $4.2 \pm 0.4$ & $9.41 \pm 1.4$ & 1.47 \\
\hline \multirow[t]{2}{*}{ PG $0052+251 \ldots \ldots$} & 780 & 1.39 & $3958 \pm 565$ & 8.59 & $6.25 \pm 0.6$ & $18.12 \pm 2.7$ & 2.03 \\
\hline & & 0.6 & $1696 \pm 283$ & 0.38 & $0.27 \pm 0.03$ & $0.81 \pm 0.1$ & \\
\hline \multirow[t]{2}{*}{ PG $0157+001 \ldots \ldots$} & 824 & 1.29 & $3832 \pm 590$ & 5.67 & $4.61 \pm 0.5$ & $7.74 \pm 1.2$ & 2.43 \\
\hline & & 0.5 & $1474 \pm 295$ & 0.4 & $0.32 \pm 0.03$ & $0.54 \pm 0.1$ & \\
\hline PG $0953+414 \ldots \ldots$ & 1239 & 1.49 & $5884 \pm 785$ & 3.14 & $5.76 \pm 0.6$ & $49.9 \pm 7.5$ & 1.05 \\
\hline PG $1012+008 \ldots \ldots$ & 973 & 1.59 & $5319 \pm 665$ & 5.01 & $5.67 \pm 0.6$ & $20.35 \pm 3.1$ & 1.54 \\
\hline PG $1049-005 \ldots \ldots$ & 2011 & 1.99 & $10484 \pm 1048$ & 5.64 & $27.28 \pm 2.7$ & $52.57 \pm 7.9$ & 0.53 \\
\hline PG $1307+085 \ldots \ldots$ & 791 & 1.39 & $4002 \pm 572$ & 6.96 & $5.21 \pm 0.5$ & $20.83 \pm 3.1$ & 1.35 \\
\hline
\end{tabular}

${ }^{\text {a }}$ These fluxes were derived by summing the flux in the HST images inside the circular aperture with the radii given in the third column.

${ }^{\mathrm{b}}$ Surface brightnesses at the edge of the NLR (those of the Seyfert galaxies were at about the same value). 

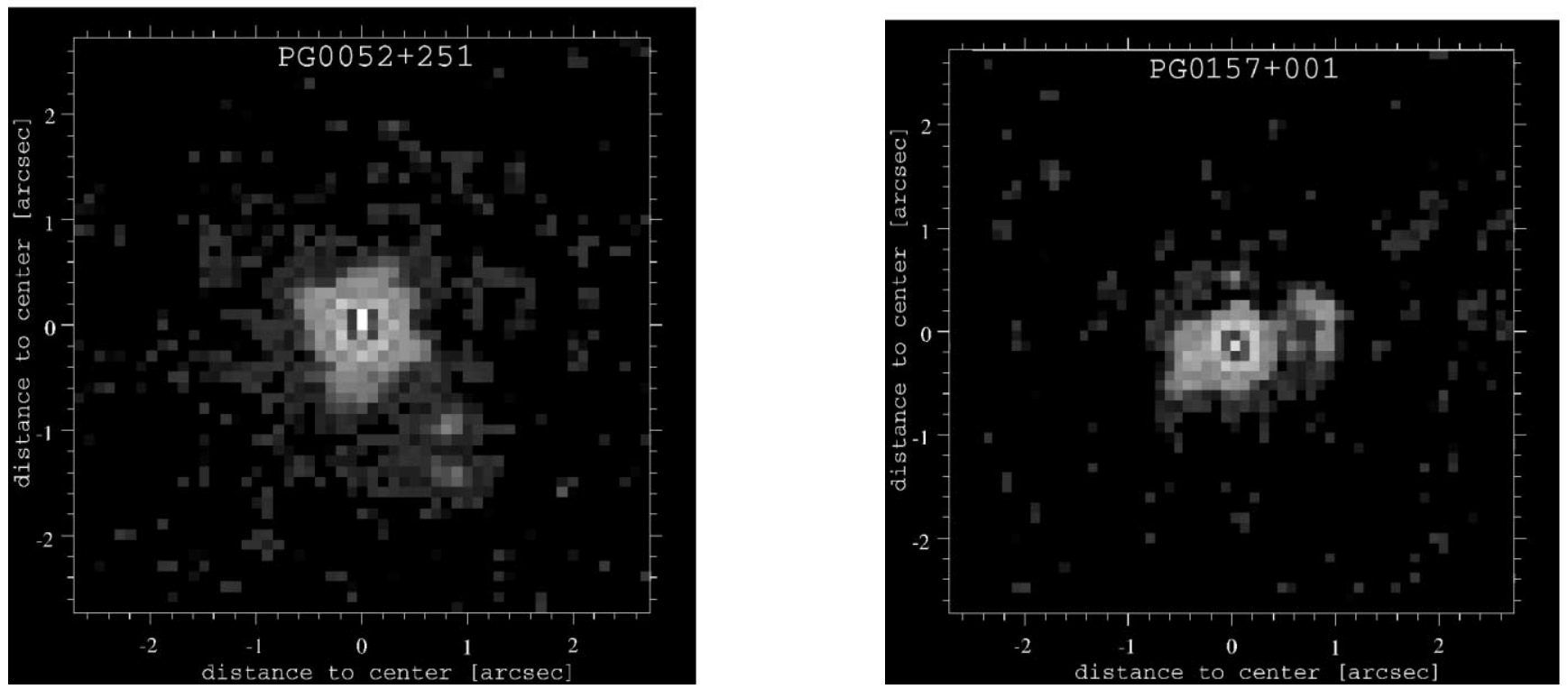

FIG. 1.-LRF images of PG $0052+251$ (left) and PG $0157+001$ (right) in [O III] $\lambda 5007$ in a logarithmic scale in false colors. North is up, and east is to the left. The tick marks are chosen such that the central pixel is at 0.0 ; major tick marks are at distances of $1^{\prime \prime}$. [See the electronic edition of the Journal for a color version of this figure.]

\section{RESULTS}

\subsection{Photometry and Structure}

For each quasar, we defined a radius of the NLR in our images at which a $3 \sigma$ level above background was reached in ring apertures. The surface brightness at that distance was typically $0.3 \%$ of the central value, thereby enclosing more than $98 \%$ of the detectable emission. The results of the photometry as well as these radii are given in Table 2 . In all cases, the NLR was largely concentrated within $2^{\prime \prime}-4$ ".

Two quasars show compact filamentary structure as shown in Figure 1. Photometry of these features (PG 0052+251: 1".5 to the south and $\sim 0$ ".8 to the west of the nucleus; PG 0157+001: $\sim 0$."4 to the north and $\sim 1^{\prime \prime}$ to the west of the nucleus) was carried out separately (see Table 2). The feature in PG 0052+251 can also be found in the broadband images of Bahcall et al. (1997) as "knots" and in a radio map from Kukula et al. (1998; classified as a possible core jet), whereas Miller, Rawlings, \& Saunders (1993) do not find a significant radio extension. The "linear" structure of PG $0157+001$ is coincident with radio features (classified as a double source with radio components on either side of the optical nucleus; see Kukula et al. 1998 and Miller et al. 1993). A close association between the NLR emission-line morphology and that of the radio emission has already been found for Seyfert galaxies (Capetti et al. 1996; Falcke et al. 1998). PG $1012+008$ and PG 0026+129 reveal some extended radio structure (Miller et al. 1993; Kukula et al. 1998), but we do not find extended emission-line features. Five out of the seven quasars are listed in Kukula et al. (1998) with maximum radio angular extents of $0.24-2 "$.

\subsection{Size as a Function of Luminosity}

An important question is how the NLR grows as one increases the luminosity of the central engine from Seyfert to quasar luminosities. For that reason, we compared the sizes and luminosities of the NLR in our quasar sample with the NLR of a sample of Seyfert 2 galaxies that we have studied earlier. This sample was observed with HST in a very similar fashion, using LRFs and imaging the [O III] emission line (Falcke et al. 1998). In Figure 2, we plot the linear sizes versus the luminosities for the Seyfert galaxies and quasars.

The number of sources is of course very limited, but with the addition of the quasars, we are probing 3 orders of magnitude in luminosity. The weighted linear least-squares fit is (weight $=\sigma^{-2}$, where $\sigma$ is the standard error in $\log R$; units of $R$ and $L$ are parsecs and ergs $\mathrm{s}^{-1}$, respectively):

$$
\log R_{\mathrm{NLR}}=(0.52 \pm 0.06) \log L_{[\mathrm{O} I \mathrm{II}]}-(18.5 \pm 2.6)
$$

with a correlation coefficient (cc) of 0.92 for 14 data points (dp). Hence, the size of the NLR seems to scale proportionally with the square root of the [O III] luminosity.

We note that for the quasars alone, a similar relation is found, when plotting the NLR size versus the $\mathrm{H} \beta$ luminosity (Fig. 2, right). With $L_{\mathrm{H} \beta}$ derived by multiplying our $L_{[\mathrm{O} \text { III] }}$ with the $\mathrm{H} \beta /$ [O III] ratios from Boroson \& Green (1992) (see Table 2), we obtain $(\mathrm{cc}=0.85,7 \mathrm{dp})$

$$
\log R_{\mathrm{NLR}}=(0.67 \pm 0.15) \log L_{\mathrm{H} \beta}-(25.5 \pm 6.3)
$$

The average $[\mathrm{O} \mathrm{III}] / \mathrm{H} \beta$ ratio of the Seyfert galaxies is 30 times larger than that of the quasars (Bergvall, Johansson, \& Olofsson 1986; Colina et al. 1987; Cid Fernandes et al. 2001). Since the Seyfert galaxies are type 2 objects, in the framework of the unified model, only a few percent of the total $\mathrm{H} \beta$ is visible. Boroson \& Green (1992) also found that $2 \%$ of the total $\mathrm{H} \beta$ flux is visible as the narrow-line component. We have thus multiplied the inferred narrow-line $\mathrm{H} \beta$ luminosities of the Seyfert galaxies by a factor of 30 to estimate their total $\mathrm{H} \beta$ luminosities, and we found that the resulting $R$ versus $L_{\mathrm{H} \beta}$ relationship is consistent with an extrapolation to lower luminosity of the relationship found for the quasars. Including both Seyfert and quasar data into a weighted linear least-square fits, 

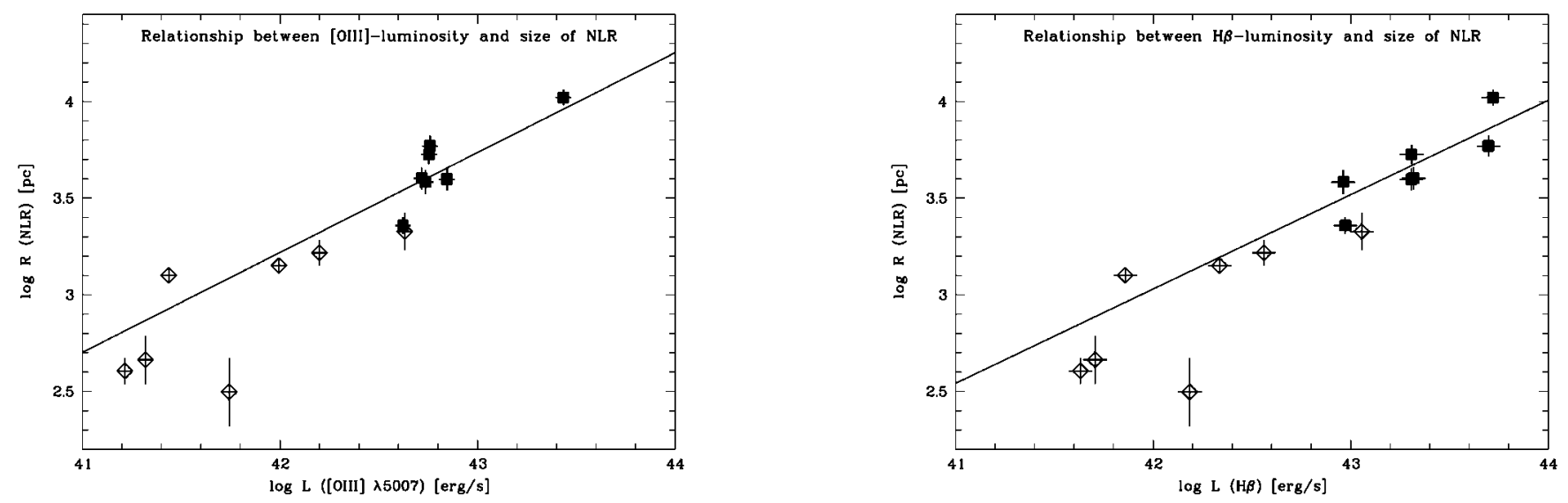

FIG. 2.-Radius of the NLR vs. the emission-line luminosity in [O III] $\lambda 5007$ (left) and in H $\beta$ (right) on logarithmic scales. The open diamonds are Seyfert galaxies; the filled squares are PG quasars. The solid lines show weighted linear fits resulting in $R \propto L_{[0 \text { m] }}^{0.52}$ (left) and $R \propto L_{\mathrm{H} \beta}^{0.49}(r i g h t)$. The error bars indicate the uncertainties in the fluxes from both the photometry and the placement of the apertures.

we obtain $(\mathrm{cc}=0.91,14 \mathrm{dp})$

$$
\log R_{\mathrm{NLR}}=(0.49 \pm 0.06) \log L_{\mathrm{H} \beta}-(17.5 \pm 3.4) .
$$

\section{DISCUSSION AND CONCLUSIONS}

We find that the NLRs of seven bright radio-quiet PG quasars are remarkably compact with typical extents of $2^{\prime \prime}-4$ ". Hence, detailed imaging of quasar NLRs requires subarcsecond resolution. Generally, the structure is relatively symmetric, in agreement with the unified scheme, which predicts a view into the ionization cones of these type 1 objects.

Two quasars exhibit compact filamentary structure like that seen for Seyfert galaxies. These structures may be related to radio outflows. This is reminiscent of the situation in many Seyfert galaxies, where radio outflows are morphologically related to the NLR. In three quasars, the radio emission is approximately as compact as the NLR, and in all five cases that can be found in the literature, the maximum angular extent is in agreement with our compact extents.

The NLR in quasars seems to be consistent with being a scaled-up version of the NLR in Seyfert galaxies. In fact, one of the Seyfert 2 galaxies, Mrk 34, has a similar radius and [O III] luminosity to the quasars and hence might be considered a "type 2 quasar." In addition, the size of the NLR seems to scale roughly with the square root of the [O III] luminosity when combining the quasar and Seyfert samples.

The latter result, however, has to be taken with some caution. For one, we have a very limited number of sources available. Especially for quasars, high-resolution images of the NLR are extremely scarce; one essentially needs the resolution of HST plus the flexibility of the LRFs to image redshifted emission lines. Second, when comparing the Seyfert 2 sample and the quasar sample, one needs to consider that, according to the unified scheme, orientation effects might bias the type 2 sample toward somewhat larger sizes. Third, the individual scatter of NLR sizes is relatively large. Fourth, the "size" of the NLR is not a well-defined quantity, being dependent on sensitivity and resolution. Nevertheless, given the large span in luminosities, none of the effects are likely to change the results significantly.

Seeking a clue to the origin of this relation, we consider a recombination line like $\mathrm{H} \beta$, rather than $[\mathrm{O}$ III], as a tracer of continuum luminosity. The ionization parameter is given by $U=$ $Q /\left(4 \pi c n_{e} R_{u}^{2}\right)\left(Q\right.$ is the rate of H-ionizing photons, and $R_{u}$ is the distance between the photoionizing source and emission-line clouds). Employing the relation $\omega Q=\alpha_{B} /\left(\alpha_{\mathrm{H} \beta} h \nu_{\mathrm{H} \beta}\right)=3.9 \times$ $10^{12} L_{\mathrm{H} \beta}$ (in cgs units; $\omega$ is the covering factor, and the recombination coefficients $\alpha_{B}$ and $\alpha_{\mathrm{H} \beta}$ are taken from Osterbrock 1989 for $T=2 \times 10^{4} \mathrm{~K}$ ) yields a relation $R_{u} \propto L_{\mathrm{H} \beta}^{0.5}$ for a given $U$, $n_{e}$, and constant $\omega$.

The slope in Figure 2 is close to the slope of $0.6 \pm 0.1$ given by Peterson (2001) for the relationship between BLR size (measured from reverberation mapping) and the continuum luminosity (at $5100 \AA$ ). McLure \& Jarvis (2002) find an even tighter correlation between the BLR radius and the $3000 \AA$ A luminosity, with both correlations being consistent with a relation of the form $R_{\mathrm{BLR}} \propto L^{0.5}$.

To first order, BLRs and NLRs exhibit similar ionization parameters commonly explained by the interaction of clouds with a quasar wind (Schiano 1986). More recently, Dopita et al. (2002) use radiation pressure dominated photoionization models to explain a constant ionization parameter (in the range of $-2.5 \leq$ $\log U \leq-2)$. At the outskirts of a NLR, $n_{e} \sim 10^{2}-10^{3} \mathrm{~cm}^{-3}$, and $U \sim 10^{-2}$ to $10^{-3}$, so that efficient [O III] emission comes from regions with $U n_{e} \approx 1$, corresponding to an ionizing photon rate $Q /\left(4 \pi R_{u}^{2}\right)=3 \times 10^{10}$ photons $\left(\mathrm{s}^{-1} \mathrm{~cm}^{-2}\right)$. Individual values of $R_{u}$ derived with this scheme are slightly larger than the measured $R$, corresponding to covering fractions $\omega \leq 1$.

The same average $[\mathrm{O} \mathrm{III}] / \mathrm{H} \beta$ ratio for Seyfert galaxies and quasars preserves the slope of the size-[O III] relation for a size- $\mathrm{H} \beta$ relation. This corroborates the explanation in terms of the ionization parameter and argues for photoionization as the main underlying process for producing narrow lines.

As pointed out by the referee, the relationship $L_{[\mathrm{O} \text { III] }}$ (or $\left.L_{\mathrm{H} \beta}\right) \propto R_{\mathrm{NLR}}^{2}$ corresponds to a constant [O III] (or $\mathrm{H} \beta$ ) surface brightness. However, we are not aware of any other physical, or instrumental, reason why this should be the case since [O III] is emitted by the whole volume filled with NLR clouds.

Clearly, more HST observations of quasars with lower [O III] luminosity are needed to test the validity of the correlation. We expect fainter [O III] quasars to have even smaller NLRs than the sources presented here, and such a program will present an observational challenge for the years to come. Nevertheless, the available data provide the first direct evidence that quasar and Seyfert NLRs are related and possibly evolve along a common luminosity-size track determined by photoionization. 
N. B. thanks T. Jürges for computer support, C. Leipski for discussions, and R. Chini for financial support. This research was also supported by STScI through grant GO 8239 to the University of Maryland and the University of Texas.

\section{REFERENCES}

Antonucci, R. R. J. 1993, ARA\&A, 31, 473

Bahcall, J. N., Kirhakos, S., Saxe, D. H., \& Schneider, D. P. 1997, ApJ, 479, 642

Bergvall, N., Johansson, L., \& Olofsson, K. 1986, A\&A, 166, 92

Boroson, T. A., \& Green, R. F. 1992, ApJS, 80, 109

Capetti, A., Axon, D. J., Macchetto, F., Sparks, W. B., \& Boksenberg, A. 1996, ApJ, 469, 554

Cid Fernandes, R., Heckman, T., Schmitt, H., González Delgado, R. M., \& Storchi-Bergmann, T. 2001, ApJ, 558, 81

Colina, L., Fricke, K. J., Kollatschny, W., \& Perryman, M. A. C. 1987, A\&A, 186, 39

Dopita, M. A., Goves, B. A., Sutherland, R. S., Binette, L., \& Cecil, G. 2002, ApJ, 572, 753

Falcke, H., Wilson, A. S., \& Simpson, C. 1998, ApJ, 502, 199

Kellermann, K. I., Sramek, R., Schmidt, M., Shaffer, D. B., \& Green, R. 1989, AJ, 98, 1195
Kukula, M. J., Dunlop, J. S., Hughes, D. H., \& Rawlings, S. 1998, MNRAS, 297, 366

McLure, R. J., \& Jarvis, M. J. 2002, MNRAS, submitted (astro-ph/0204473) Miller, P., Rawlings, S., \& Saunders, R. 1993, MNRAS, 263, 425

Osterbrock, D. E. 1989, Astrophysics of Gaseous Nebulae and Active Galactic Nuclei (Mill Valley: University Science Books)

Perlmutter, S., et al. 1999, ApJ, 517, 565

Peterson, B. M. 2001, preprint (astro-ph/0109495)

Pogge, R. W. 1988, ApJ, 328, 519

-. 1989, ApJ, 345, 730

Schiano, A. V. R. 1986, ApJ, 302, 81

Schmidt, M., \& Green, R. F. 1983, ApJ, 269, 352

Schulz, H. 1988, A\&A, 203, 233

Storchi-Bergmann, T., Mulchaey, J. S., \& Wilson, A. S. 1992, ApJ, 395, L73 